\title{
Early and late selection in partial report: Evidence from degraded displays
}

\author{
D. J. K. MEWHORT, ELIZABETH E. JOHNS, and SHANNON COBLE \\ Queen's University, Kingston, Ontaraio, Canada
}

\begin{abstract}
In studies of iconic memory using the bar-probe task, subjects see a brief display of target letters and are probed by an arrow to report one of them. According to the classic early-selection account, subjects use the probe to select material for perceptual analysis from a precategorical (iconic) memory, but according to late-selection theories, subjects first identify the letters and then use the probe to select one letter for report from the set of categorized items. Pashler (1984) based his test for the locus of selection on a manipulation of display quality in previewed displays. He presented a target for $200 \mathrm{msec}$ and then added a probe, together with the target, for an additional $\mathbf{1 5 0}$ msec. Reducing the target's stimulus quality increased response latency. If the subjects identified the characters before the probe appeared and then selected an item for report, the clarity of the original array should not have affected response latency. Hence, Pashler concluded that his subjects used the probe to select from a precategorical store (early selection). Pashler's experiment did not force subjects to rely on memory of the target; hence, although his experiment documented a situation in which subjects used early selection, it did not rule out late selection in studies of information persistence. We replicated Pashler's findings and, using his logic, showed that when subjects are forced to rely on memory of the target, they select from a categorized store.
\end{abstract}

In studies of visual persistence using the bar-probe task, subjects are shown a display of letters tachistoscopically, and an arrow then indicates one letter to report. Thus, the subjects' task is to select and to report one letter on the basis of a spatial cue. When the probe follows the target without overlapping it temporally, the subjects are forced to rely on memory of the target, and accuracy of report decreases as the time between the target and the probe increases (Averbach \& Coriell, 1961).

Two broad classes of theory have been suggested to explain how subjects select an item in the bar-probe task; these classes may be distinguished by how they define the data that are selected. In early-selection theories on the one hand, subjects use the probe as a perceptual guide; they store a precategorical representation of the letter array (named iconic memory by Neisser, 1967) and use the probe to select part of that representation for perceptual analysis. The reduction in accuracy associated with a delayed probe is attributed to the loss through decay of the feature data that are required for perceptual analysis to occur.

The research was supported by Grant AP-318 from the Natural Sciences and Engineering Research Council of Canada. The paper is based on a presentation at a joint meeting of the Experimental Division of the Canadian Psychological Association and the Experimental Psychology Society (U.K.) in Oxford, England, July 1987. We thank D. Feldman-Stewart for help in running the experiments. Address for correspondence: D. J. K. Mewhort, Department of Psychology, Queen's University, Kingston, Ontario K7L 3N6, Canada (Electronic mail: Doug@vip.psyc.queensu.ca).
In late-selection theories, on the other hand, subjects identify the letters without reference to the probe and store a categorized representation of the target instead of the precategorical image (icon) suggested by early-selection accounts. The subjects then use the probe to select one item from the categorized store for report. The reduction in accuracy associated with a delayed probe is attributed to the loss of information that the subject needs in order to retrieve the item from the correct location.

There is another distinction between early- and lateselection theories: In early-selection theories, it is usually claimed that our capacity to identify characters is limited, whereas in late-selection theories it is usually claimed that identification is automatic (see Shiffrin, 1976). In the present paper, however, we will focus on the first criterion-the kind of data selected.

\section{TESTS FOR THE LOCUS OF SELECTION IN PARTIAL REPORT}

Several kinds of test have been advanced to distinguish early from late selection in partial-report tasks. Here we will discuss three kinds: tests based on efficiency of selection, on the fate of unattended material, and on errors of selection. The experimental work reported thereafter involved a fourth kind of test-namely, one based on manipulation of stimulus quality.

\section{Selection Efficiency}

The first kind of test examines the stimulus dimensions that permit effective selection. Here, an advantage of par- 
tial over whole report is the measure of success in selection. Initial evidence supported the early-selection theories by suggesting that, although subjects can select by perceptual dimensions such as location, they are unable to select by symbolic dimensions such as alphanumeric class. As Merikle (1980, Experiments 3-5) has documented, however, in the initial experiments selection was confounded with response organization because partial report was compared with whole report in a between-subjects design. When Merikle equated organizational uncertainty in the two conditions by mixing partial- with whole-report trials in a within-subjects design, subjects were able to select by alphanumeric class, a result consistent with the late-selection view.

\section{The Influence of Unselected Material}

In a second kind of test, the influence of unselected material on report of the selected material is examined. If subjects select material from an uncategorized display for perceptual analysis, as the early-selection theories suggest, the unselected material should remain in uncategorized form, and its structure should not affect report of the selected material. Hence, evidence that structure of the unselected material affects report of selected material favors late-selection theories.

Tests based on the influence of unselected material are often inconclusive because proponents of early selection can take refuge in the possibility that attention has lapsed momentarily and that, as a result, nominally unattended material has been selected during the lapse of attention. Consider, however, an early study by Mewhort (1967). He presented two eight-letter pseudowords arranged in two rows and used a tone to cue the subjects to report letters from one of the two rows, a close variant of Sperling's (1960) classic task. The pseudowords were of either zero- or fourth-order approximation to English and were arranged in one of four configurations: two zero-order sequences, a zero-order sequence above a fourth-order sequence, a fourth-order sequence above a zero-order sequence, or two fourth-order sequences. The partial-report cue was presented immediately after the offset of the target.

The number of letters reported correctly was determined by the familiarity of both the rejected and the selected pseudowords taken together-the number of letters reported correctly from the designated row reflected the average familiarity of the two pseudowords. Because the structure of the rejected material affected report of the selected material, the subjects could not have used an early-selection strategy. Moreover, because the effective familiarity of fourth-order material requires left-right analysis (CRYSTEMP loses its familiarity when printed backwards: PMETSYRC; see Wolff \& Mewhort, 1986), it is implausible to attempt an explanation based on a momentary lapse of attention.

\section{Errors of Selection}

A third kind of test is based on the kind of error associated with the bar-probe task; early-selection theories suggest that errors should share visual features with the target. Yet, as Mewhort, Campbell, Marchetti, and Campbell (1981) have documented, subjects do not make errors in a bar-probe task because of feature-level confusions, but because they tend to report characters from unprobed positions in the display (see also Mewhort, Butler, FeldmanStewart, \& Tramer, 1988). Moreover, the decrease in accuracy associated with a delayed cue is complemented by an increase in intra-array errors. Thus, the pattern of errors is not consistent with the early-selection view.

In reply, an early-selection theorist might suppose that subjects identify some items nonselectively, without waiting for the probe, and, as a result, that they have a subset of the items in memory when the probe appears. If, when data are lost through decay, subjects report from the pool of items in memory instead of basing their reports on the features remaining at the probed location, one can explain the large number of intra-array errors without abandoning early selection and the idea of a fading icon.

For different reasons, both Mewhort, Marchetti, Gurnsey, and Campbell (1984) and van der Heijden, Schreuder, de Loor, and Hagenzieker (1987) have argued that such a position predicts that extra-array confusions ought to take an inverted- $U$ pattern as the probe is delayed. Because extra-array errors increased linearly with probe delay instead of taking an inverted- $U$ pattern, Mewhort et al. (1984) concluded in favor of a late-selection account. Van der Heijden et al. (1987) argued, however, that only a subset of extra-array errors should reflect visual confusion and that those errors took an inverted- $U$ across probe delay. Hence, they concluded in favor of a modified earlyselection view. Unfortunately, the method that van der Heijden et al. used to separate visual confusions from extra-array errors was flawed; they used a restricted set of letters in the display, and they overlooked the possibility that subjects might restrict the range of responses when guessing an item at the probed location on the basis of the letters identified from other locations. In effect, in their calculation of chance they assumed early selection, and as a result, their experiment did not provide a fair test of late selection.

\section{EXPERIMENT 1}

In the present paper, we consider a fourth kind of test, one in which the target's stimulus quality is manipulated (Pashler, 1984). The logic is that degrading the stimulus should slow identification of uncategorized material but should not slow retrieval from a pool of identified characters.

Pashler's (1984) procedure was as follows: On each trial, subjects were shown four copies of two letters (" $A$ " and ' $E$ "); the eight letters were arranged randomly within two rows of four. An arrow marked one character, and the task was to indicate as rapidly as possible whether the arrow marked an " $A$ " or an "E." The target was clear or degraded, and this factor was combined factorially with three timing conditions. In the first, the target and the arrow were shown concurrently. In the second, the arrow was presented for $200 \mathrm{msec}$; then the target was added 
to the display. In the third, the target was presented for $200 \mathrm{msec}$; then the arrow was added to the display. In all cases, the target and arrow appeared together for $150 \mathrm{msec}$. The dependent measure was response time, measured from the point at which both the target and the probe were available.

Degrading the stimulus increased response latency, and the increase was independent of the timing conditions. The interpretation of the concurrent and probe-first conditions is straightforward: Reducing display quality increased response latency by slowing subjects' perceptual analysis of the character probed, a result compatible with both early and late selection. In the target-first condition, however, subjects had an opportunity to complete stimulus identification before the probe appeared. If subjects used the opportunity to complete stimulus identification-as late-selection theory suggests-degrading the stimulus should not have affected response time. Accordingly, Pashler (1984) concluded that his subjects must have delayed categorization until the probe appeared and then used the probe to select precategorical data for perceptual analysis. Thus, his subjects used an early-selection strategy even when a late-selection strategy was possible.

In Pashler's (1984) paradigm, the target and the probe were always shown together for $150 \mathrm{msec}$; the experiment did not force subjects to rely on memory of the target. Thus, the experiment documented one situation in which subjects preferred an early-selection strategy, but it did not rule out late selection when subjects must rely on memory of the target. As a result, it did not address selection from visual memory or the status of iconic memory.

In the present experiments, we extended Pashler's (1984) work by applying his logic to the situation in which subjects rely on memory of the target. The first experiment was designed to validate our implementation of his paradigm.

\section{Method}

Subjects. The subjects were 12 students recruited through a summer student-employment service and paid for their services. All subjects reported normal or corrected-to-normal vision.

Apparatus. The stimuli were presented on a display monitor (Tektronix Model 604) equipped with P4 phosphor. The monitor's viewing area subtended a visual angle of about $9.18^{\circ}$ (horizontal) $\times$ $7.42^{\circ}$ (vertical). The display monitor was controlled by a PDP-11/23 computer housed in an adjacent room. The computer was equipped with three microswitches. One was used to start the trials; the remaining two were used to indicate the subject's choices.

Stimuli. Each target array was constructed by arranging four copies of an uppercase " $A$ " and four copies of an uppercase " $E$ " randomly in two equal rows. A different random arrangement was used on each trial. The two rows were centered within the monitor's viewing area and subtended a visual angle of approximately $1.63^{\circ}$ (horizontal) $\times 0.78^{\circ}$ (vertical).

Each character was defined in a $5 \times 7$ matrix and subtended a maximum visual angle of $0.28^{\circ} \times 0.21^{\circ}$. Degraded characters were generated by deleting at random about $30 \%$ of the dots normally used to define the character. The letter "A," for example, is defined by 18 dots. To degrade an "A," we removed 6 dots at random; we used a different random selection each time that we used the character.
The probe was an arrow constructed from a seven-dot line with two two-dot fins attached at the top for an upward-pointing arrow or at the bottom for a downward-pointing arrow. The arrow pointed down at the target from the row above when the target was in the top row; it pointed up at the target from the row below when the target was in the bottom row.

Procedure. At the start of each trial, a dim fixation dot was presented at the center of the monitor's viewing area. When ready, the subject initiated the trial by pushing a button. About $10 \mathrm{msec}$ after the subject pushed the start button, the stimuli appropriate to the condition were presented on the monitor. The subject responded by pushing one button to indicate " $A$ " and another to indicate " $E$ "; the computer recorded both the response and its latency. After recording the response, the computer calculated the dot positions needed for the next trial and presented the fixation point to initiate the next trial. Room illuminance was 3.5 lux, and the luminance of the monitor's screen was about $0.5 \mathrm{~cd} / \mathrm{m}^{2}$. Letters were whiteblue (about $4.4 \mathrm{~cd} / \mathrm{m}^{2}$ ) on a dark screen.

Timing conditions. The target's duration was either 150 or $450 \mathrm{msec}$, and the probe's duration was either 150 or $450 \mathrm{msec}$. The target and the probe were aligned temporally at their offset. Thus, when their durations were equal, the target and probe were concurrent for either 150 or $450 \mathrm{msec}$. When the durations were unequal, the longer stimulus lead the shorter one by $300 \mathrm{msec}$, but both appeared together for $150 \mathrm{msec}$. Response latency was measured from the time at which both the target and the probe were available.

Design. Each subject was tested on 512 trials, eight replicates for the factorial combination of four variables: (1) target duration (150 or $450 \mathrm{msec}$ ), (2) probe duration (150 or $450 \mathrm{msec}$ ), (3) probe position (eight positions based on two rows of four characters), and (4) display quality (degraded or not). The order in which the conditions were administered was randomized independently for each subject.

\section{Results}

Dependent measures. Accuracy and latency scores for each trial were summarized as a function of target duration, probe duration, and display quality. Each of the resulting eight cells contained data for 64 trials per subject.

As noted earlier, it has been useful in previous barprobe experiments to classify errors by type, in particular to tally intra- and extra-array errors separately (see, e.g., Mewhort, Marchetti, Gurnsey, \& Campbell, 1984). Unfortunately, because only two responses were permitted in the study, it was not possible to classify errors by type.

All eight distributions of the latency scores were skewed to the right. To explore the potential bias associated with such distributions, we calculated several dependent measures. The dependent measures included (1) mean latency of correct responses, (2) mean latency regardless of accuracy (the score Pashler used), (3) median latency of correct responses, and (4) median latency regardless of accuracy. In each case, we calculated the four measures using untrimmed data, data trimmed by rejecting trials with a latency greater than $1,500 \mathrm{msec}$, and data trimmed by rejecting trials with a latency less than $150 \mathrm{msec}$ or greater than 1,500 msec. All 12 measures revealed essentially the same results. We report the mean across subjects of the median latency calculated, regardless of accuracy, on the untrimmed scores.

Response latency. Response latency is shown in Table 1 , as a function of target duration, probe duration, 
Table 1

Median Response Latency (in Milliseconds) and Accuracy (Percentage Correct) as a Function of Target Duration, Probe Duration, and Display Quality in Experiment 1

\begin{tabular}{|c|c|c|c|c|c|c|}
\hline \multirow{2}{*}{\multicolumn{2}{|c|}{ Duration }} & \multicolumn{4}{|c|}{ Display Quality } & \multirow[b]{3}{*}{ Target-Probe Relation } \\
\hline & & \multicolumn{2}{|r|}{ Clear } & \multicolumn{2}{|c|}{ Degraded } & \\
\hline Target & Probe & RT & $\%$ Correct & RT & \% Correct & \\
\hline 150 & 150 & 630 & 94 & 643 & 93 & target and probe concurrent \\
\hline 150 & 450 & 601 & 93 & 625 & 94 & probe leads/overlaps target \\
\hline 450 & 150 & 522 & 93 & 553 & 91 & target leads/overlaps probe \\
\hline 450 & 450 & 637 & 97 & 654 & 94 & target and probe concurrent \\
\hline
\end{tabular}

and display quality. Providing a preview of the probe (the probe-leads condition) reduced response latency; that reduction resulted in a large interaction of target and probe duration $[F(1,11)=88.36, p<.001]$. A comparable effect appeared in Pashler's (1984) data.

Degrading the target array increased response latency by about $22 \mathrm{msec}[F(1,11)=25.78, p<.001]$. There were no significant interactions involving display quality; in particular, a 300-msec preview of the target (the target-leads condition) did not eliminate the effect of degrading the target. Thus, the present results clearly replicate Pashler's (1984) main findings.

Accuracy. The accuracy data are summarized in Table 1 also. Accuracy was not affected by display quality $[F(1,11)=1.44, p>.20]$, and there were no significant interactions involving that variable. Likewise, accuracy was equivalent across the eight display positions $[F(7,77)=0.85]$. There was, however, a strong interaction of target and probe duration: Accuracy was highest in the 450-msec exposure concurrent condition $[F(1,11)$ $=19.57, p<.01]$.

\section{Discussion}

Degrading the target reduced response latency, even in the target-leads condition. If subjects had used the lead time to identify the material-as late-selection theories suggest-selection would have been made from a pool of identified items, and the quality of the preidentification data could not have affected response latency. We conclude, therefore, that our subjects selected from an uncategorized store.

The present results are very similar to Pashler's (1984) findings. We conclude, therefore, that we have a valid implementation with which to explore the paradigm.

\section{EXPERIMENT 2}

In Pashler's (1984) experiment (and in our replication), the target and the probe were present together for $150 \mathrm{msec}$; hence, the subjects could delay stimulus identification until the probe appeared. Although the finding demonstrates that subjects do not automatically start to categorize items at stimulus onset, it does not prove that they cannot do so. When subjects are forced to rely on memory of the target, for example, they may elect a strategy of identifying and then selecting, because they prefer to store the target in a categorized rather than an uncategorized memory.

In the second experiment, the stimuli and general procedure were the same, except that the onset of the probe followed the offset of the target. The change in procedure forced subjects to rely on memory of the target and thus permitted us to consider both selection from visual memory and the nature of visual persistence.

\section{Method}

Subjects. The subjects were 14 students recruited as before.

Procedure. The procedure was essentially the same as in Experiment 1 . The exception was the timing of the target relative to the probe: The probe always followed immediately after the target's offset.

\section{Results}

The data were treated in the same fashion as in Experiment 1 , and we report the same dependent measure. Both accuracy and response latency are shown in Table 2, as functions of target duration, probe duration, and display quality.

Response latency. In Experiment 1, there was a strong interaction of target duration with probe duration-an interaction reflecting better performance in the probe-leads condition. In Experiment 2, the probe always followed the target, and the corresponding interaction was not significant $[F(1,13)<1.0]$. Increasing the target's duration increased response latency by about $63 \mathrm{msec}[F(1,13)=$ 17.64, $p<.01$ ].

In Experiment 1, degrading the stimulus increased response latency by about $22 \mathrm{msec}$. In Experiment 2, by contrast, the same manipulation increased latency by about

Table 2

Median Response Latency (in Milliseconds) and Accuracy (Percentage Correct) as a Function of Target Duration, Probe Duration, and Display Quality in Experiment 2

\begin{tabular}{|c|c|c|c|c|c|}
\hline & & \multicolumn{4}{|c|}{ Display Quality } \\
\hline \multicolumn{2}{|c|}{ Duration } & \multicolumn{2}{|c|}{ Clear } & \multicolumn{2}{|c|}{ Degraded } \\
\hline Target & Probe & RT & \% Correct & RT & $\%$ Correct \\
\hline 150 & 150 & 693 & 76 & 703 & 68 \\
\hline 150 & 450 & 698 & 70 & 697 & 70 \\
\hline 450 & 150 & 749 & 75 & 761 & 73 \\
\hline 450 & 450 & 757 & 78 & 775 & 76 \\
\hline
\end{tabular}


$10 \mathrm{msec}$, but the increase was far from significant $[F(1,13)$ $=1.11, .25<p<.35]$. There were no interactions with the display quality (all $F \mathrm{~s}<1.0$ ). A null effect of display quality is, of course, the result anticipated on the basis of late-selection theories.

Accuracy. Accuracy in Experiment 1 was above $90 \%$ correct in all cells; accuracy in Experiment 2 averaged about $73 \%$ correct. Increasing the target duration increased accuracy slightly (from about $71 \%$ to about $75 \%$ ) $[F(1,13)=5.75, p<.05]$, and degrading the target decreased accuracy slightly (from about $75 \%$ to about $71 \%)[F(1,13)=6.97, p<.02]$. Accuracy was about the same for letters from the top row as it was for letters from the bottom ( $72 \%$ and $74 \%$, respectively); accuracy was better for the material in the two center positions as opposed to the two outer positions $(76.5 \%$ and $70 \%$, respectively).

\section{Discussion}

Pashler (1984) found that degrading the stimulus increased response latency even when subjects had plenty of time to process the target. He correctly took that result as evidence for early selection-his subjects used the probe to select from an uncategorized representation of the target. We argued, however, that his experiment did not demonstrate that visual memory must take an uncategorized form, because it did not force subjects to select from a stored representation of the target.

In Experiment 2, the probe followed the offset of the target, thereby forcing the subjects to rely on their memory of the target. We found no evidence that reducing the display quality increased response latency. We must conclude that our subjects did not select from a precategorical (i.e., iconic) memory; instead, they used a strategy of identifying and then selecting.

Accuracy in Experiment 2 was much lower than it was in Experiment 1. This lower level of accuracy was not unexpected. In previous studies of visual memory in which subjects elected a strategy of identifying and then selecting, accuracy was affected by limited spatial abilities (e.g., Mewhort \& Campbell, 1978; Mewhort et al., 1981). Specifically, subjects often erred by reporting a letter adjacent to the letter probed. Such errors suggest a retrieval failure caused by misalignment of the subject's representation of the target with the subject's representation of the probe or by spatial confusion within the stored target array itself (cf. Butler, Mewhort, \& Tramer, 1987). The forced-choice technique used in the present experiments does not permit us to confirm that pattern of errors directly. Nevertheless, if spatial errors did occur with the frequency observed in the earlier studies, the present subjects must have erred on a goodly number of trials by attempting to retrieve the wrong letter. Such localization errors explain why accuracy was $20 \%$ lower in Experiment 2 than it was in Experiment 1, even though the display duration and other visual conditions-the determinants of the identification component of the task-were identical.

\section{EXPERIMENT 3}

By forcing subjects to rely on their memory of the target in Experiment 2, we eliminated the effect of stimulus quality on response latency. A null effect is not strong evidence, however. Hence, we thought it prudent to repeat the demonstration: in Experiment 3, we included both a probe-leads and a target-leads condition. As in Experiment 2 , the target and the probe were never present together.

\section{Method}

Subjects. The subjects were 15 undergraduates recruited as before. Procedure. The procedure was similar to that of Experiments 1-2. The target array and the probe were the same as before, except that their durations were fixed at $150 \mathrm{msec}$. On half of the trials, the target was presented first, and the probe appeared at the target's offset; for the remaining trials, the probe was presented first and the target appeared at the probe's offset. As before, the target array was degraded on half of the trials.

Design. Each subject received 256 trials, eight replications for the factorial combination of three variables: (1) display quality (degraded or not), (2) target-probe order (target first or probe first), and (3) position probed (eight positions, based on two rows of four characters). The order in which the conditions were administered was randomized independently for each subject.

\section{Results}

We collapsed the data into four cells, one for each combination of display quality and target-probe order; each cell was based on 64 trials per subject. Our latency measure was based, as before, on the mean across subjects of the median scores.

Response latency. As is shown in Table 3, targetprobe order was a major determinant of response latency; when the probe was presented ahead of the target, subjects were about 100 msec faster than they were when the order was reversed $[F(1,14)=68.08, p<.001]$. Furthermore, when the probe was first, degrading the target increased response latency by about $31 \mathrm{msec}[F(1,14)=$ $5.04, p<.05]$, a replication of the corresponding result in Experiment 1. When the order was reversed, by contrast, degrading the stimulus did not affect latency $[F(1,14)$ $=0.16]$, a replication of the null result found in Experiment 2. The interaction of display order with display quality, however, was not significant $[F(1,14)=1.31$, $p<.30]$.

Accuracy. As is shown in Table 3, when the probe was first, accuracy was about $93 \%$-the level of accuracy that was found in Experiment 1 when the target and probe were concurrent. When the target was first, accuracy fell

Table 3

Median Response Latency (in Milliseconds) and Accuracy (Percentage Correct) as a Function of Target-Probe Order and Display Quality in Experiment 3

\begin{tabular}{cccccc}
\hline & \multicolumn{3}{c}{ Display Quality } \\
\cline { 2 - 3 } \cline { 5 - 6 } $\begin{array}{c}\text { Target-Probe } \\
\text { Relation }\end{array}$ & RT & \% Correct & & RT & \% Correct \\
\cline { 2 - 5 } Target first & 739 & 74 & & 744 & 71 \\
Probe first & 623 & 94 & & 654 & 92 \\
\hline
\end{tabular}


to $72 \%$-about the same level as in Experiment 2. The difference was highly significant $[F(1,14)=108.66$, $p<.001]$. Degrading the target reduced accuracy about $2.5 \%[F(1,14)=3.54, .05<p<.10]$. As in Experiment 2 , accuracy was about the same for material in both rows, but accuracy for material in the central positions was slightly better than that for material from the outer positions (85.5\% and $79.5 \%$, respectively).

\section{Discussion}

Our interpretation of the results is the same as it was before. When the probe was presented first, the subjects used it to focus on a narrow part of an uncategorized representation. When the target appeared, they started to identify the relevant letter. Identification of a degraded target was slower than identification of an intact target.

When the target was presented first, in contrast, subjects were forced to resort to a different strategy-namely, that of identifying and then selecting, as they identified the material and stored it in a categorized buffer. Although identification may have been slower for degraded than it was for intact material, this fact could not be documented, because the timer was not started until the probe arrived. When the probe arrived (and the timer started), the subjects used the probe to retrieve one of the items from the postcategorical store. Because subjects had plenty of time to identify the material before the timer started, degrading the stimulus did not affect response latency.

\section{EXPERIMENT 4}

The pattern of results from Experiment 3 confirms subjects' use of a late-selection strategy when sampling from visual memory. The evidence, however, is weaker than one would like. Although we twice found a degrading effect when the probe was first (in Experiments 1 and 3) and twice found a null effect of display quality when the target was first (in Experiments 2 and 3), we acknowledge that the data are not sufficiently compelling. In Experiment 3, for example, degrading the stimulus significantly lengthened response time with the probe first but had no effect with the target first, a pattern suggesting an interaction of order with quality. Paradoxically, the interaction was not significant, an anomaly that weakens the evidence by suggesting that subjects were unable to sustain a stable strategy. In addition, changing display order significantly altered accuracy: Accuracy was much lower when the target was first than when the probe was first. Response latency is a dubious index of processing difficulty when accuracy is low, especially when chance is as high as $\mathbf{5 0 \%}$. The difficulty is that a large number of the response times may reflect the time to make a lucky guess, not the time to process the material.

In Experiment 4, we attempted to correct some of the difficulties in our data by considering response latency separately for a subset of the correct trials on which accuracy was very high. To do so, we asked subjects to provide a confidence rating for each response. We expected that correct responses for which subjects expressed high confidence would be sufficiently accurate to permit a clear interpretation of the latency data. In addition, to give subjects a solid chance to develop a stable strategy, we manipulated target-probe order between-subjects.

\section{Method}

Subjects. The subjects were 18 students who were recruited as before.

Procedure. The procedure was based on the earlier experiments. We simplified the display, however, by filling the two rows of four letters with two pairs of EE and AA; the position of each pair was determined randomly on successive trials.

In addition to the timed $A$ versus $E$ response, the subjects were required to indicate their confidence in each decision. Following the timed response, they reported their confidence on a three-point scale, on which 1 indicated low confidence (guessing), 3 indicated high confidence (sure), and 2 was a middle value.

Design. There were two groups of subjects; one group was tested with the probe first, and the other was tested with the target first. For both groups, the probe and target durations were $150 \mathrm{msec}$, and the probe followed immediately after the offset of the target or vice versa. Each subject was tested on 256 trials-16 replications for the factorial combination of two variables: display quality (degraded or not) and position probed (eight positions, based on two rows of four characters). The order in which the conditions were administered was randomized independently for each subject.

\section{Results}

Mean accuracy and mean response latency for both correct responses and errors are shown in Table 4, as a function of the subjects' confidence that the response was correct. The table also shows the proportion of trials associated with each level of confidence.

Accuracy. In the target-first group, accuracy on lowconfidence trials was at chance $(52.2 \%$; chance is $50 \%$ ). Mean latency was about $90 \mathrm{msec}$ shorter on low-confidence error trials than it was on low-confidence correct trials. It appears, therefore, that the low-confidence data are contaminated by a number of fast guesses. On high-confidence trials, by contrast, accuracy was a respectable $91 \%$, and the latency on high-confidence correct trials was slightly shorter than the latency on high-confidence error trials. Hence, we can accept the latency for high-confidence cor-

Table 4

Accuracy (Percentage Correct) and Response Latency (in Milliseconds) for Both Correct Reports and Errors as a Function of Subjects' Confidence and Target-Probe Order in Experiment 4

\begin{tabular}{|c|c|c|c|c|}
\hline \multicolumn{2}{|c|}{ Confidence } & \multirow[b]{3}{*}{$\%$ Correct } & \multirow{2}{*}{\multicolumn{2}{|c|}{ Mean Latency }} \\
\hline \multirow{2}{*}{$\begin{array}{c}\text { Confidence } \\
\text { Rating }\end{array}$} & \multirow[b]{2}{*}{$\%$ Trials } & & & \\
\hline & & & Correct & Errors \\
\hline \multicolumn{5}{|c|}{ Target First } \\
\hline 1 & 12 & 52 & 855 & 765 \\
\hline 2 & 27 & 78 & 805 & 727 \\
\hline 3 & 61 & 91 & 634 & 665 \\
\hline \multicolumn{5}{|c|}{ Probe First } \\
\hline 1 & 6 & 52 & 442 & 387 \\
\hline 2 & 13 & 85 & 622 & 379 \\
\hline 3 & 81 & 85 & 612 & 400 \\
\hline
\end{tabular}




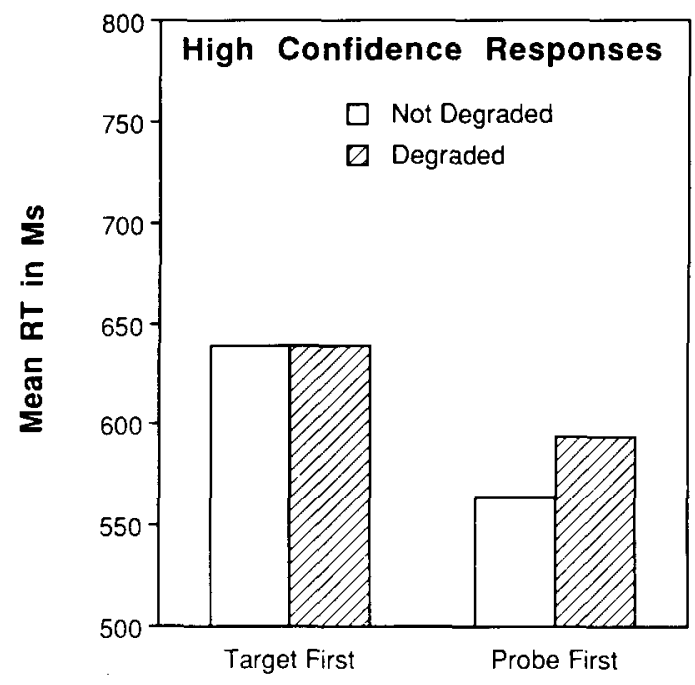

Figure 1. Response latency as a function of target quality and target-probe order for high-confidence trials in Experiment 4.

rect responses in the target-first group as a measure of processing difficulty.

In the probe-first group, accuracy on low-confidence trials was also at chance $(52.2 \%$ correct). Mean latency on low-confidence error trials was about $55 \mathrm{msec}$ shorter than on low-confidence correct trials, a pattern suggesting that the low-confidence data are contaminated by fast guesses. On high-confidence trials, the difference in latency on error and correct trials was even larger (over $200 \mathrm{msec}$ ). Thus, in marked contrast to the target-first case, the high-confidence data are likely to be contaminated by fast guesses and, therefore, to consist of latencies shorter than the processing time required. Accordingly, we deleted responses with a latency of $150 \mathrm{msec}$ or faster. Most of the deleted responses were from 1 subject.

Latency. For each subject, we calculated the mean latency for the correct responses to which the subject had given a high confidence rating greater than $100 \mathrm{msec}$. The results are summarized in Figure 1. Note, however, that because the mean latency for the probe-first condition is likely less than the time actually required, it is not directly comparable to the corresponding mean for the target-first condition.

For the target-first group, there was no evidence that degrading the target slowed response latency $[F(1,8)<$ 1.0], a result that corroborates the results of Experiments 2 and 3. For the probe-first group, in contrast, degrading the stimulus array lengthened response latency by about $30 \mathrm{msec}[F(1,8)=20.24, p<.01]$. The interaction of order with degradation was significant $[F(1,16)$ $=4.42, p<.05$ ].

\section{GENERAL DISCUSSION}

The results can be summarized as follows: When the probe was presented ahead of, or overlapping the target, the subjects elected an early-selection strategy; that is, they used the probe to select part of a precategorical representation for identification. Accordingly, they took longer to identify a target that had been presented in a degraded form as opposed to one that was presented clearly. When the subjects were forced to rely on memory of the target, by contrast, they elected a late-selection strategy; that is, they identified the material, stored it in a postcategorical memory, and used the probe to retrieve a categorized item. Because the subjects had enough time to identify the material before the probe arrived (and the timer started), degrading the stimulus did not affect response latency.

What do the results mean for the concept of iconic memory? The originators of the partial-report paradigm believed that they were studying subjects' use of a precategorical store (e.g., Averbach \& Coriell, 1961; Sperling, 1960). The present results, and the tests cited earlier, all contradict the theory of the precategorical store: In spite of the immense popularity of the idea in introductory texts, subjects in partial-report studies of visual memory do not select from a precategorical store; they select from a postcategorical representation.

By arguing against the traditional view of iconic memory, we do not deny that subjects have a precategorical (iconic) representation of a visual display. Rather, our point is that methods once thought to give access to that representation do not do so. If one wants to study the initial representation of a stimulus, new methods are needed (such as the method explored by Di Lollo, 1980).

We prefer a two-buffer account for performance in probe tasks. Consider, for example, the study by Mewhort et al. (1984). They compared masking and direct degrading (of the sort used in the present study) in a postexposure bar-probe experiment. By delaying the mask and by adjusting the number of dots removed from the target display, Mewhort et al. equated the number of errors for both techniques. Even though subjects produced the same number of errors, the kind of error depended on the technique: Masking yielded mostly intra-array errors, whereas direct degrading produced mostly extra-array errors. Mewhort et al. argued that direct degrading reduced the quality of feature information and, thereby, reduced accuracy by making identification difficult. As a result, the bulk of the errors with direct degrading were extra-array errors. By contrast, they argued that masking scrambled position information primarily at the postcategorical level and, thereby, reduced accuracy by making localization difficult. As a result, the bulk of the errors with masking were intra-array errors. Whatever the merits of Mewhort et al.'s specific interpretation, it is difficult to imagine how one could account for the pattern of results in terms of a single precategorical buffer.

Our conclusion is that subjects elect an early- or a lateselection strategy, depending on the demands of the task (for further recent evidence see Butler, Mewhort, \& Browse, 1991, Experiment 3). Such flexibility conflicts with ideas often thought to separate the early- and late- 
selection views (see, e.g., Duncan, 1980). Following Broadbent (1958), proponents of early selection have typically assumed that subjects have a limited capacity to carry out stimulus identification. Accordingly, subjects are thought to retain a precategorical store and to use the probe to designate which item(s) to identify. But, our target-thenprobe results suggest that capacity limitations for item identification are not strained with displays such as those in the present experiments. If capacity limits are not strained, why would subjects adopt an early-selection strategy, the strategy we claim for the probe-then-target condition?

The answer is that the probe-then-target condition encourages early selection. Late selection implies that subjects identify all the material and then sort the identified items to retrieve the correct one. But, the task was to identify one item as fast as possible. With the probe first, subjects can use the probe to locate the general position of the target and then note the local position and the identity of enough features to identify a character. With instructions to respond as rapidly as possible, it is clear that late selection simply offers no advantages over early selection in the probe-then-target condition.

When the probe follows the target, by contrast, subjects must rely on memory of the target, and the relative costs of early and late selection are changed. The barprobe task taxes both identification and localization processes. Whereas the capacity idea traditionally focuses on limitations on identification, we assume that subjects elect a strategy by considering the costs associated with both identification and localization. With an early-selection strategy in the target-then-probe case, subjects would have to remember the local position and the identity of virtually all visual features in the display until the probe arrives. We assume that it is easier to remember the position of one letter in a string of four than it is to remember the position of the many separate features that constitute four letters in an uncategorized store. Our assumption implies that subjects can reduce their memory load by categorizing the material-it is to the subjects' advantage to store a categorized representation and to use the probe to guide retrieval from that store.

We have argued that subjects elect a late-selection strategy when they must rely on memory. But, as two reviewers have pointed out, when one attends to an object in the real world, it rarely disappears, and, therefore, we rarely have to depend on memory. It would seem to follow that selection in the real world consists of early selection. Thus, even if we should succeed in replacing the iconicmemory account with a late-selection account, we would be achieving a pyrrhic victory, inasmuch as iconic memory research tells us little about selection in the real world.

The problem here is that the argument ignores eye movements and the role a postcategorical representation plays in integrating information across saccades. The facts are that the eyes sample the world, that information is integrated across fixations (see, e.g., Blanchard, Pollat- sek, \& Rayner, 1989; Inhoff, 1990; Rayner, McConkie, \& Zola, 1980), and that, as McConkie and Zola (1979) note, "visual features of the type which specify the difference between upper- and lowercase letters are not integrated across fixations during reading" (p. 221). In short, readers do not use a precategorical visual memory but depend on an abstract postcategorical representation (see Inhoff \& Tousman, 1990). When viewed from a lateselection perspective, the real-world relevance of iconicmemory research lies in the possibility that one can study the postcategorical representation without the complications introduced by eye movements.

\section{REFERENCES}

Averbach, E., \& Coriell, A. S. (1961). Short-term memory in vision. Bell System Technical Journal, 40, 309-328.

Blanchard, H. E., Pollatsek, A., Rayner, K. (1989). The acquisition of parafoveal word information in reading. Perception \& Psychophysics, 46, 85-94.

Broadbent, D. E. (1958). Perception and communication. London: Pergamon.

Butler, B. E., Mewhort, D. J. K., \& Browse, R. A. (1991). When do features migrate? A boundary condition for feature-integration theory. Perception \& Psychophysics, 49, 91-99.

Butler, B. E., Mewhort, D. J. K., \& Tramer, S. C. (1987). Location errors in tachistoscopic recognition: Guesses, probe errors, or spatial confusions. Canadian Journal of Psychology, 41, 339-350.

Di LoLlo, V. (1980). Temporal integration in visual memory. Journal of Experimental Psychology: General, 109, 75-97.

DUNCAN, J. (1980). The locus of interference in the perception of simultaneous stimuli. Psychological Review, 87, 272-300.

INHOFF, A. W. (1990). Integrating information across eye fixations in reading: The role of letter and word units. Acta Psychologica, 73, 281-297.

INHOFF, A. W., TousmaN, S. (1990). Lexical integration across saccades in reading. Psychological Research, 52, 330-337.

MCConkIE, G. W., ZoLA, D. (1979). Is visual information integrated across successive fixations in reading? Perception \& Psychophysics, 25, 221-224.

MerikLe, P. M. (1980). Selection from visual persistence by perceptual groups and category membership. Joumal of Experimental Psychology: General, 109, 279-295.

MEwhorT, D. J. K. (1967). Familiarity of letter sequences, response uncertainty, and the tachistoscopic recognition experiment. Canadian Journal of Psychology, 21, 309-321.

Mewhort, D. J. K., Butler, B. E., Feldman-Stewart, D., \& Tramer, S. (1988). "Iconic memory,"' location information, and the bar-probe task: A reply to Chow (1986). Joumal of Experimental Psychology: Human Perception \& Performance, 14, 729-737.

Mewhort, D. J. K., Campela, A. J. (1978). Processing spatial information and the selective-masking effect. Perception \& Psychophysics, 24, 93-101.

Mewhort, D. J. K., Campbell, A. J., Marchetti, F. M., CAmpBELL, J. I. D. (1981). Identification, Iocalization, and "iconic" memory: An evaluation of the bar-probe task. Memory \& Cognition, 9, 50-67.

Mewhort, D. J. K., Marchetti, F. M., Gurnsey, R., CampBELL, A. J. (1984). Information persistence: A dual-buffer model for initial visual processing. In H. Bouma \& D. Bouwhuis (Eds.), Attention and performance $X$ : Control of language processes (pp. 287-298). Hillsdale, NJ: Erlbaum.

Neisser, U. (1967). Cognitive psychology. Appleton-Century-Crofts.

PAshler, H. (1984). Evidence against late selection: Stimulus quality effects in previewed displays. Journal of Experimental Psychology: Human Perception \& Performance, 10, 429-448. 
RAYNer, K., MCConkie, G. W., \& Zola, D. (1980). Integrating information across eye movements. Cognitive Psychology, 12, 206-226.

SHIFFrIN, R. M. (1976). Capacity limitations in information processing, attention, and memory. In W. K. Estes (Ed.), Handbook of learning and cognitive processes: Vol. 4. Attention and memory (pp. 177236). Hillsdale, $\mathrm{NJ}$ : Erlbaum.

SPERLING, G. (1960). The information available in brief visual presentations. Psychological Monographs, 74, (11, Whole No. 498).

van der Heuden, A. H. C., SChreuder, R., De Loor, M., \& Hagen-
ZEIKER, M. (1987). Early and late selection: Visual letter confusions in a bar-probe task. Acta Psychologica, 65, 75-89.

WolfF, P., \& MEWHORT, D. J. K. (1986). Building higher-order units in tachistoscopic identification: A test of two models. Psychological Research, 48, 79-85.

(Manuscript received November 20, 1989; revision accepted for publication March 19, 1991.) 